\title{
Percepción del mercado costarricense acerca del uso cerchas prefabricadas con madera de plantaciones forestales y unidas con placas metálicas
}

\section{Perception of Costa Rican market about the use of prefabricated trusses with wood from forest plantations and joined with metal plates}

\author{
Monzerrath Rivera-Tenorio ${ }^{1} \bullet$ Diego Camacho-Cornejo² (iD) $\bullet$ Roger Moya²
}

\begin{abstract}
In housing construction, the most efficient structures to support the loads coming from roofs correspond to the trusses. When it comes to manufacturing them with wood, an important point of these are the joints. Currently the use of metal plates has been introduced for these unions, however, the market still knows little about them and its possibility of use in wooden houses. Therefore, this study reports a first qualitative survey by means of a survey stratified of the use of wooden trusses with metal plates fabricated with plantation wood in Costa Rica. It was found that $100 \%$ of respondents consider wood as a viable material for construction, for storing carbon dioxide and for being a renewable resource. In addition, most consider that the wood from trees growing in plantations in Costa Rica is of good quality. However, only $28 \%$ prefer the use of trusses as a structural element in roofs and $40 \%$ agree that the use of the metal plate is a good technological option. According to this first market survey of the wooden truss with metal plate, $80 \%$ of respondents would be willing to acquire it; giving it a price range between $₫ 45000$ to $₫ 50000$ per truss.
\end{abstract}

Key words: Wooden construction, trusses, plantation wood, joints.

1. Consultor Independiente; Cartago, Costa Rica; r.monse05@gmail.com

2. Escuela de Ingeniería Forestal, Tecnológico de Costa Rica; Cartago, Costa Rica; dicamacho@tec.ac.cr;_rmoya@tec.ac.cr 
En construcción de viviendas, las estructuras más eficientes para soportar las cargas provenientes de techumbres corresponden a las cerchas. Cuando se trata de fabricarlas con madera, un punto importante de estas son las uniones. Actualmente se ha introducido la utilización de placas metálicas dentadas para estas uniones, sin embargo, el mercado aún conoce poco de ellas y su posibilidad de utilización en casas. Por lo que, este estudio reporta un primer sondeo cualitativo por medio de una encuesta estratificada por público objetivo, del uso de cerchas de madera con placas metálicas dentadas fabricadas con madera de plantación en Costa Rica. Fue encontrado que el $100 \%$ de los encuestados considera a la madera como un material viable para la construcción, por almacenar dióxido de carbono y ser un recurso renovable. Además, el $90 \%$ de los encuestados, considera que la madera de las plantaciones nacionales es de buena calidad. Sin embargo, sólo un $28 \%$ prefiere la utilización de las cerchas como elemento estructural en techumbres y el $40 \%$ coincide en que el uso de la placa es una buena opción tecnológica. Según este primer sondeo de mercado de la cercha de madera con placa metálica, el $80 \%$ de encuestados estaría dispuesto a adquirirla; dándole un rango de precio entre $\$ 45000$ a $\$ 50000$ por cercha.

Palabras clave: Construcción con madera, cerchas, madera de plantación, uniones.

\section{Introducción}

En construcción con madera la resolución de una gran variedad de cubiertas de edificaciones es y ha sido ampliamente resuelta mediante cerchas de madera, desde aquellas de pequeña luz ejecutadas con madera aserrada y pocos miembros, hasta grandes luces con mayor número de elementos y madera laminada [1]. En general, existen una infinidad de tipos y formas de cerchas, para adaptarse a las necesidades de un proyecto específico [2]. Sin embargo, cuando se trata de estructuras utilizadas para soportar las cargas provenientes de techumbres en forma triangular y luces de hasta $10 \mathrm{~m}$, se demuestra que las tipologías más eficientes corresponden a las cerchas denominadas Howe, Pratt y Fink. El diseño de un sistema de cerchas del tipo triangular, son mayormente utilizadas para luces pequeñas, porque así las cargas se transmiten a la cercha en puntos específicos [3]. En el diseño se busca que cada cercha trabaje de forma independiente respecto de las otras [4].

Por otra parte, para el logro de una adecuada construcción con madera, un aspecto base es la realización de las uniones entre los diferentes elementos que conformarán la estructura y que son los sectores más vulnerables en este tipo de edificación [5]. La resolución de estas uniones es una de las particularidades del diseño para estructuras en madera. En otros materiales no resulta complicado realizar uniones rígidas, pero en madera este tipo de uniones es de difícil ejecución [6]. Diferentes tipos de conectores son utilizados para las uniones en cerchas, entre las que destaca clavos, tornillos y placas metálicas [7]. Según Rammer [8], se ha evidenciado que, al utilizar conectores metálicos, es posible obtener una mayor resistencia en la cercha, por lo que comúnmente se llaman placas de refuerzo. En Norte América las placas de metal como principal unión en las cerchas de madera, gozan de un registro probado sobresaliente en rendimiento y durabilidad [9]. Sin embargo, este tipo de uniones y sus respectivas resistencias apenas se conocen en las estructuras construidas con especies tropicales [10].

Costa Rica cuenta con madera de varias especies proveniente de plantaciones forestales para abastecer la demanda de madera, a la cual se le han estudiado sus valores de esfuerzos admisibles para grados estructurales [11]. En este país, recientemente han surgido maderas de plantaciones forestales [12], entre las que destaca Gmelina arborea y Tectona grandis. Estas maderas han estado en el mercado en los últimos 15 años, y por ello son las maderas de las cuales se tiene mayor conocimiento sobre su procesamiento industrial y factores de su comercialización [13]. Sin embargo, en general para las especies de plantación, es necesario desarrollar aún más experiencia con el fin de aumentar su uso en el mercado y así aumentar el área a reforestar [14]. Serrano y Moya [13] presentan un análisis histórico sobre la comercialización de la madera en Costa Rica, donde indican que durante la mayor parte del siglo pasado la madera aserrada era comercializada sin mayor criterio técnico; se vendía sin dificultad en el mercado y no se necesitaba de técnicas avanzadas de comercialización. En el caso de la madera de plantación -debido a su variabilidad y que las dimensiones de las trozas son bajas - necesita volúmenes apropiados para desarrollar un mercado, tener buen desempeño en los procesos industriales y desde luego un mercado establecido [11].

Además, en Costa Rica es necesario incursionar en nuevos mercados para crear productos, que se adapten a los actuales diseños arquitectónicos [15]. Como lo es la construcción tipo liviana y prefabriado o tipo "framing" en su término inglés [12], la cual consiste en el uso de un mayor volumen de madera con construcción de marco ligero, con facilidad y rápidez de montaje [1]. A pesar de la información disponible del uso de las maderas de plantación, aún se carece del conocimiento 
de las propiedades estructurales de estas en los procesos de construcción tipo liviana (framing) como las cerchas prefabricadas [12].

La poca tecnificación de la madera proveniente de las plantaciones tropicales, unido al poco conocimiento que manejan ingenieros y arquitectos del uso de esta madera, provoca que no utilicen este material en sus diseños [16]. Asimismo, la falta de agresividad de mercadeo en el sector de comercialización de la madera, son factores que han contribuido al debilitamiento del uso de la madera en la construcción en la actualidad en Costa Rica. Entonces el utilizar madera estructural, específicamente en una cercha con madera proveniente de plantación, representa una oportunidad en el mercado y así introducirla en usos de mayor valor agregado. Además de lograr la máxima resistencia posible de la madera en la cercha, por la utilización de conectores metálicos tipo placas.

Otro aspecto importante es conocer muy bien el tipo de madera que se va a producir y el segmento o nicho de mercado al cual se dirige la producción [17]. Ya que, aunque se hagan diseños apropiados de productos, muchos de estos pueden llegar a fracasar sino se ha hecho un estudio adecuado de la percepción del producto [18]. Un estudio de mercado juega un papel importante, que incluye efectuar la encuesta $y$, la elaboración de estadísticas para analizar las tendencias en el consumo, con el fin de prever la cantidad de productos y la localización de los mercados más rentables de un determinado tipo de bien o servicio [19]. Entonces, la investigación de mercados es parte de la investigación aplicada, ya que persigue recopilar datos de hechos reales o de comportamientos reales con el fin de generar tendencias, crear escenarios decisorios y entregar fuentes de información sólidas para ser consideradas como una base informativa, que permita mitigar la toma de decisiones en todo tipo de contexto donde se desenvuelve el ser humano [20].

Por ende, el estudio de mercado se da por etapas, donde Hernández, Fernández y Baptista [21] indican que, la primera etapa es la exploratoria y/o descriptiva; y en esta no se debe concluir ni generalizar, es necesario solo citar porcentualmente el comportamiento de las variables con algunas medidas de tendencia central, para la posterior toma de decisiones. Esta etapa descriptiva del estudio, debe contemplar variables tales como: opiniones, percepciones, motivaciones y actitudes [22]. Por ejemplo, una variable clave en el campo del mercado, es el precio: que para ciertos estudios será considerada como independiente (influencia en las ventas) y, para otros, como dependiente, como en el ámbito de la producción [19].

Por ello el presente trabajo, pretende conocer una primera perspectiva de como el mercado costarricense percibe el uso de la madera de las plantaciones nacionales en la construcción, así como la posible introducción de la cercha de madera construida con placas metálicas.

\section{Materiales y métodos}

Se realizó un enfoque cualitativo [21] de percepción del mercado acerca del uso cerchas de madera en casas de habitación. La investigación cualitativa, es útil para analizar variables tales como opiniones, percepciones, motivaciones y actitudes; y estas variables son de interés en el campo del mercado [22]. Por lo que se aplicó al potencial uso y mercado de las cerchas de madera. La cercha es una estructura ampliamente utilizada en el país en casas de habitación construida en la actualidad de metal, con los consecuentes problemas ambientales que esto conlleva. Entonces el estudio de la percepción del mercado permitirá obtener información sobre el conocimiento técnico; un nuevo diseño en madera y la posible adquisición de una cercha de madera más tecnificada en relación a la que se usaba en el pasado. En la realización de este enfoque cualitativo de mercado fue seguido el diagrama de flujo presentado en la figura 1.

\section{Definición de las fuentes de información}

Se definió el Público Objetivo (muestra) que pueden usar o promover el uso de las cerchas de madera, por lo que fueron estratificados en dos tipos: fuentes primarias y fuentes secundarios. Se estableció como

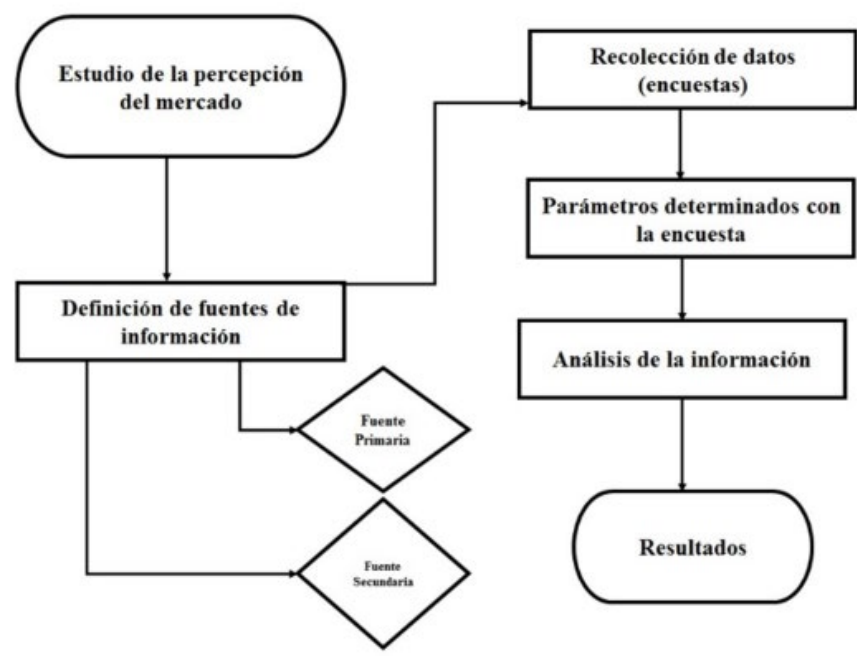

Figura 1. Diagrama de flujo para el estudio de mercado en el consumo de madera en construcción y adquisición de cercha de madera.

Figure 1. Wood for building usage and wood trusses purchase flow diagram. 
fuentes primarias, a los diseñadores estructurales de las construcciones civiles de Costa Rica. Fue seleccionado estos por ser los primeros en poder recomendar el uso potencial de la madera y específicamente la cercha. En este grupo se contemplan a ingenieros civiles, ingenieros en construcción y arquitectos. Como fuentes secundarias se definió a los potenciales compradores de la cercha, entre los que se incluyen: comerciantes de madera y las empresas constructoras de casas de madera. También al ser cerchas con fin de utilización en casas de interés social también profesionales y/o empresas de entes financieros, y fueron incluidos en las fuentes secundarias.

Se recolectó la información (nombre personal y/o nombre de la empresa y correo electrónico) para todo el país, a partir de los registros de las siguientes entidades:

- Registros de la Oficina Nacional Forestal (ONF)

- Registros del Instituto Costarricense de la madera (ICOMADERA)

- Páginas amarillas y/o redes sociales.

\section{Recolección de datos por medio de encuestas al público objetivo}

De la información de los registros, se hizo una selección aleatoria de 30 personas, para encuestar (tanto de fuentes primarias como secundarias). La información base que se obtuvo del público objetivo con preguntas abiertas, fueron: nombre personal, nombre de la empresa y correo electrónico. A partir de ello en esta segunda etapa se llevó a cabo el levantamiento de encuestas, por medio de un formulario con preguntas abiertas y de selección múltiple. El diseño del formulario se creó con la finalidad de obtener la información y los datos de: conocimiento de uso de la madera en construcción, conocimiento de la madera de plantaciones nacionales, consumo de cerchas, productos sustitutos de la cercha en techumbres, conocimiento en el uso de placas metálicas dentadas y sondeo de precios.

Este formulario fue diseñado con las herramientas: formulario de Google y Google Analytics, las cuales se utilizan para aplicar y analizar las respuestas de encuestas vía web [23]. El formulario se envió vía correo electrónico a las personas y empresas de interés. Se dio un tiempo prudencial de 15 días para ser contestada. $Y$ al tener las respuestas se procedió a extraer de Google Analytics los gráficos y tablas para su análisis.

Análisis de la información: De los datos que se obtuvieron en las encuestas se realizó un análisis por distribución de los encuestados por profesión y/u ocupación, opinión de las principales razones para construir con madera, opiniones de la madera proveniente de plantaciones forestales en Costa Rica, según encuesta de mercado, preferencia de uso de la cercha en comparación con los principales productos para techumbres, razones para la utilización de cerchas de madera, conocimiento y opinión de la utilización de conectores de placa metálica dentada en la fabricación de cerchas de madera y rangos de precios para venta de cerchas de madera, con placa metálica dentada. Dicha información fue presentada mostrando el porcentaje de opinión en relación a la cantidad de ítems totales contestadas.

\section{Diseño de la cercha propuesta}

La configuración de las cerchas se diseñó tomando en cuenta que el número de ángulos de corte en la madera aserrada fuera mínimo y que la cantidad de madera a utilizar por cercha sea poca cantidad, con la finalidad que fuese competitiva en el mercado. El diseño seleccionado fue la cercha estándar tipo howe, donde hubo una repetición de los mismos ángulos y longitudes en los diferentes montantes y diagonales. También las cerchas fueron simétricas, es decir, una pieza central (montante central) y a los lados hubiera simetría en ángulos y longitudes (figura 2). La cuerda inferior tuvo una longitud de $720 \mathrm{~cm}$ (para una luz de ensayo de $600 \mathrm{~cm}$ y un alero de $60 \mathrm{~cm}$ ). Además, las longitudes de las piezas que conformaron la parte interna de la cercha fueron: el montante central con 108,0 cm de largo (se consideró una pendiente del $30 \%$ ), los montantes laterales 49,5 $\mathrm{cm}$ y los diagonales de $151 \mathrm{~cm}$ (figura 2). En lo referente a la fabricación de las cerchas, las cuerdas superiores y la cuerda inferior de esta fueron fabricadas con madera aserrada con dimensiones de $5,0 \mathrm{~cm}$ en ancho, $10,0 \mathrm{~cm}$ en peralte y $250,0 \mathrm{~cm}$ de longitud, y para los montantes y diagonales las dimensiones fueron de $5,0 \mathrm{~cm}$ en ancho, $5,0 \mathrm{~cm}$ en peralte y $250,0 \mathrm{~cm}$ de longitud (figura 2).

\section{Placas metálicas en el diseño}

En el diseño se utilizaron placas metálicas dentadas de la marca ALPINE (figura 3). Estas placas cumplían con los requisitos y estándares recomendados por TPI (por sus siglas en inglés Truss Plate Institute) (2014), las cuales indican que estás poseen un tratamiento anticorrosivo de al menos $1 \mathrm{~mm}$ de espesor nominal y son fabricadas de acero. Las placas poseen las siguientes características: 1,11 clavos $\mathrm{cm}^{-2}$, un peso de $0,833 \mathrm{gr} \mathrm{cm}^{-2}$ y un espesor de $1,0 \mathrm{~mm}$. Además, cumplieron con los siguientes requisitos mecánicos mínimos: límite de fluencia de $2400 \mathrm{~kg} \mathrm{~cm}^{-2}$, resistencia a la rotura de $3400 \mathrm{~kg} \mathrm{~cm}^{-2}$, tensión admisible de $1000 \mathrm{~kg} \mathrm{~cm}^{-2}$ a $1200 \mathrm{~kg} \mathrm{~cm}^{-2}$. Dos dimensiones de placas dentadas fueron utilizadas: (i) Una placa de $4 \mathrm{~cm}$ de ancho $\times 10 \mathrm{~cm}$ de longitud, en las juntas compuestas de dos piezas, específicamente las uniones 1 y 5 de la figura 1. (ii) Para las uniones donde se unieron tres piezas (es decir, las uniones 2, 3 y 4 de la 


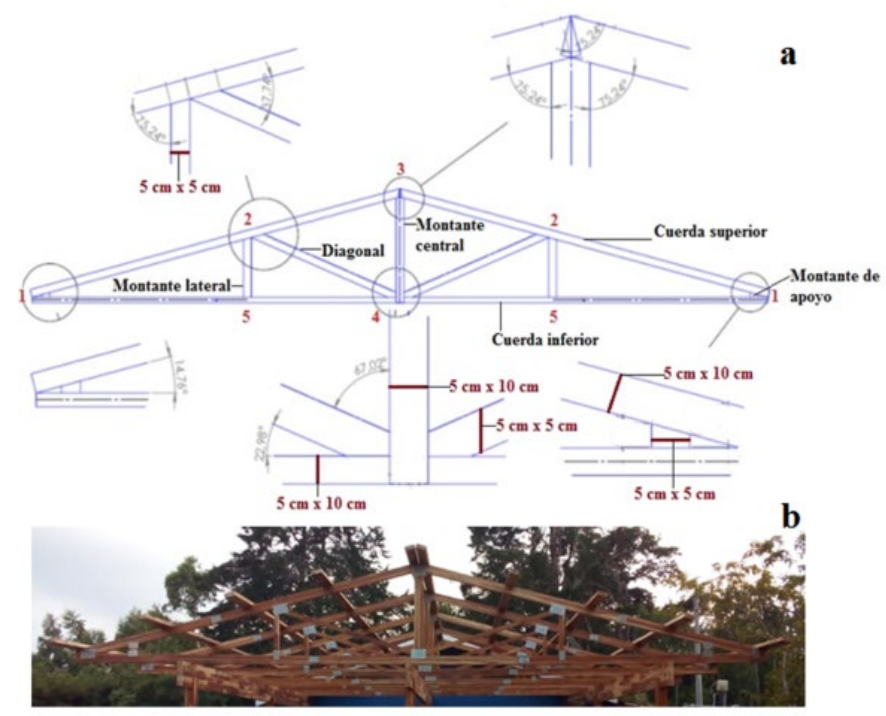

Figura 2. Diseño, partes y dimensiones; así como los tipos de uniones presentes en las cerchas tipo howe (a). Utilización de la cercha (b).

Figure 2. Design, parts and dimensions; as well as the types of joints present in howe trusses. Use of truss (b)

figura 1) se utilizaron placas de $10,0 \mathrm{~cm}$ de ancho y 15,0 $\mathrm{cm}$ de longitud.

La cantidad de placas por cerchas fueron de 22 placas de las dimensiones de $4 \mathrm{~cm}$ de ancho $\times 10 \mathrm{~cm}$ de longitud, correspondiente a 11 uniones, con dos placas por unión, una por cada lado de la cercha. En tanto que de las placas de $10,0 \mathrm{~cm}$ de ancho $\times 15,0 \mathrm{~cm}$ de longitud, se usaron 8 placas por cercha (con una distribución de 4 uniones, con dos placas por unión y a cada lado de la cercha).

\section{Resultados}

\section{Tipo de profesionales}

El análisis de la información, se basó en las encuestas de mercado contestadas, donde el nivel de respuesta fue de 36,67 \%. En primer lugar, el $50 \%$ de los encuestados correspondió a Ingenieros Civiles, un 20 \% Arquitectos, un $10 \%$ Ingenieros Industriales, un $10 \%$ Ingeniero en Construcción y un $10 \%$ Comerciante de Madera específicamente dueños de aserraderos y constructores/ vendedores de cabañas totalmente en madera (figura 4).

\section{Percepción de la madera como material}

En el análisis propiamente de los resultados de la encuesta se tiene que el $100 \%$ de los encuestados

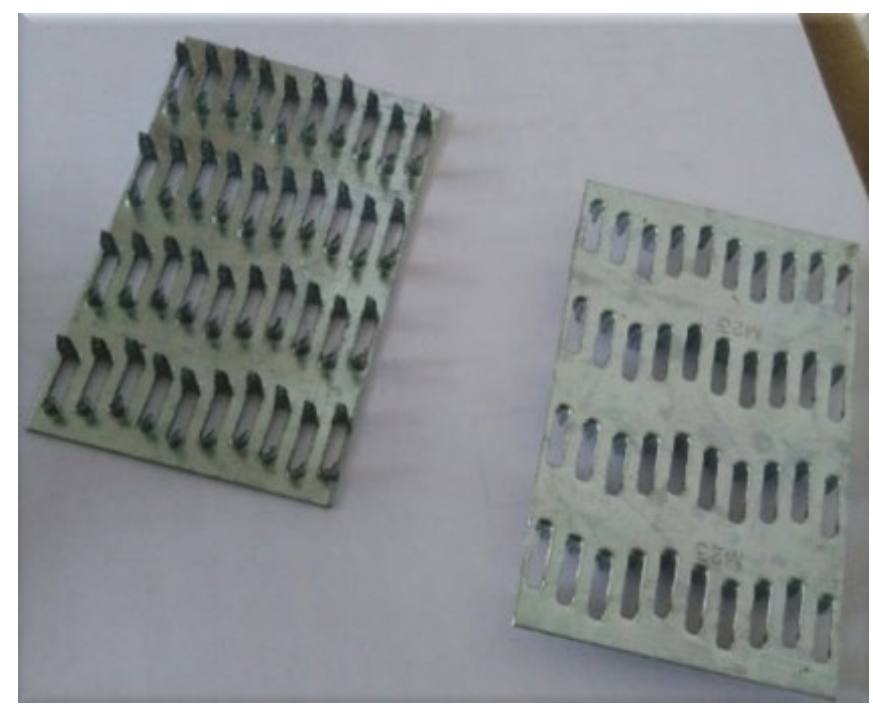

Figura 3. Placas metálicas con dimensiones de $4 \times 10 \mathrm{~cm}$.

Figure 3. Toothed metal plates with dimensions of $4 \times 10 \mathrm{~cm}$.

considera a la madera como un material viable para la construcción. Las razones principales para construir con madera fueron resumidas en 10 razones por sus altos porcentajes (cuadro 1). La razón que encabeza la lista es que la madera almacena dióxido de carbono $\left(\mathrm{CO}_{2}\right)$. Lo cual también es una de las principales razones para construir con madera en el ámbito mundial.

\section{Percepción de la madera de plantaciones forestales por el mercado}

En la encuesta de mercado se buscó conocer la opinión de los encuestados de la madera nacional y la proveniente de plantaciones forestales. En esto se obtuvo una amplia variabilidad de opiniones, donde se va desde los que la consideran madera de buena calidad, hasta la hipótesis de que no son las idóneas para construcción (figura 5).

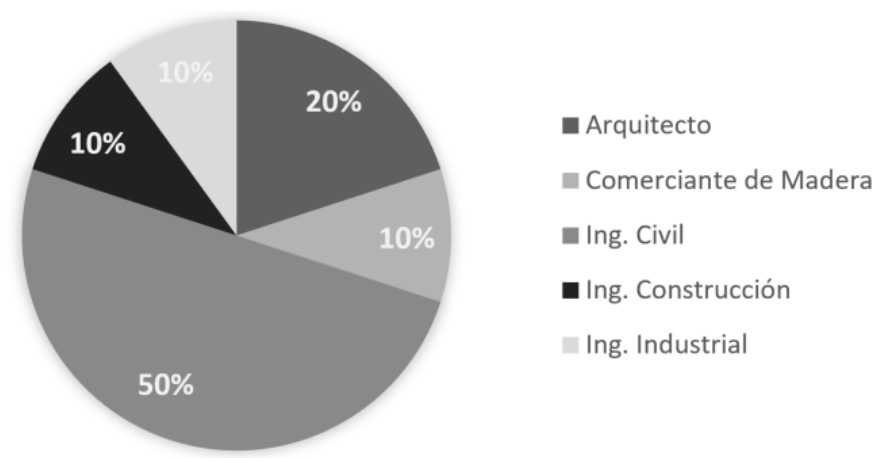

Figura 4. Distribución de encuestados según profesión u ocupación.

Figure 4. Distribution of respondents according to profession or occupation. 
Cuadro 1. Distribución de las principales razones para construir con madera, según encuesta de mercado.

Table 1. Distribution of the main reasons to build with wood, according to market survey.

\begin{tabular}{|cc|}
\hline $\begin{array}{c}\text { Razón para construir con } \\
\text { madera }\end{array}$ & \begin{tabular}{c} 
Porcentaje de respuesta \\
\hline Eficiencia estructural
\end{tabular} \\
\hline Estética & 10,7 \\
\hline Almacena $\mathrm{CO}_{2}$ & 10,7 \\
\hline Confort & 17,9 \\
\hline Versatilidad de diseño & 10,7 \\
\hline
\end{tabular}

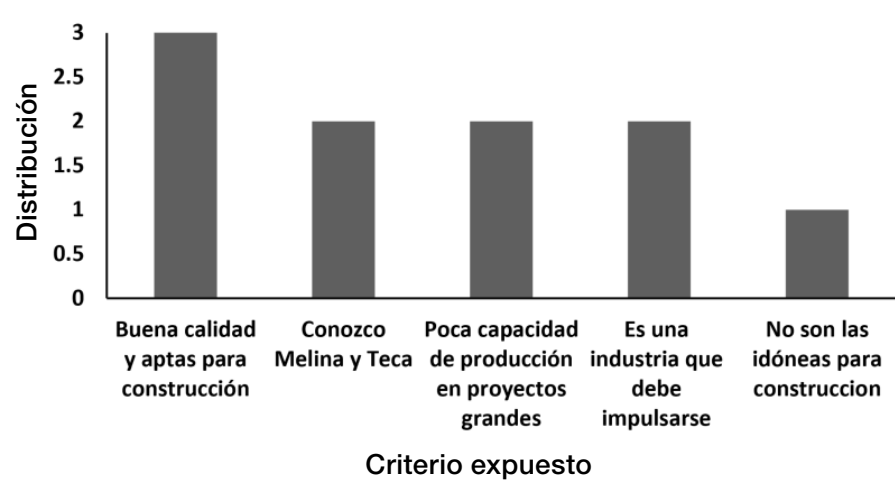

Figura 5. Distribución de opiniones de la madera proveniente de plantaciones forestales, en Costa Rica, según encuesta de mercado.

Figure 5. Distribution of opinions of wood from forest plantations, in Costa Rica, according to market survey.

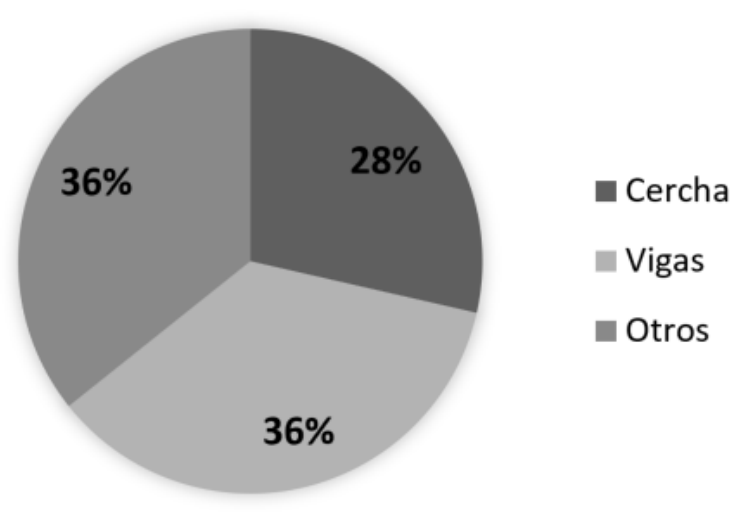

Figura 6. Distribución de la mejor resolución de techumbres para casas, en Costa Rica, según encuesta de mercado.

Figure 6. Distribution of the best resolution of roofs for houses, in Costa Rica, according to market survey.

\section{Percepción de la cercha de madera}

Para conocer la tendencia del uso de la cercha en el mercado nacional, se consultó acerca de cuál era según la opinión de los encuestados, la mejor solución para cubiertas o techumbres en la construcción de casas. Para ello se obtuvo que un $36 \%$ considera mejor la utilización de las vigas, otro $36 \%$ prefiere la utilización de otros elementos como las piezas en artesones de 5 $\mathrm{cm} \times 12 \mathrm{~cm}$ y un $28 \%$ la utilización de la cercha (figura 6).

\section{Percepción de la cercha de madera}

Para conocer la tendencia del uso de la cercha en el mercado nacional, se consultó acerca de cuál era según la opinión de los encuestados, la mejor solución para cubiertas o techumbres en la construcción de casas. Para ello se obtuvo que un $36 \%$ considera mejor la utilización de las vigas, otro $36 \%$ prefiere la utilización de otros elementos como las piezas en artesones de 5 x $12 \mathrm{~cm}$ y un $28 \%$ la utilización de la cercha (figura 6).

El cuadro 2, indica las principales razones de importancia del porque se utilizaría la cercha en la construcción de casas. La opinión del mercado acerca de este tipo de estructura su ventaja de capacidad de soporte del techo ante las cargas de viento a los cimientos. Otra importante respuesta y relacionado al aumento del rendimiento de la madera en troza es el aprovechamiento de caberías, luego le sigue otros aspectos como mayor amplitud a la casa, brinda rigidez a la estructura, soporta cargas mayores con menos material y se puede prefabricar. Finalmente, con menor cantidad de respuestas y entonces las de menor importancia para el mercado es que la cercha le brinda amarre a la estructura, permite mayor ventilación, soporte para los clavadores, brinda protección a nivel estructural; y en su construcción es práctica, barata y liviana.

\section{Conocimiento del mercado acerca de las placas metálicas dentadas}

A partir de los datos de la encuesta de mercado se evidencia que un $90 \%$ de las personas encuestadas conoce del uso de placas metálicas en la construcción de cerchas. Donde la principal opinión de los encuestados acerca de su uso es que el uso de la placa es una buena opción tecnológica e ingenieril. Además, en las opiniones se obtienen como ventajas que agiliza y reduce el espacio de la fabricación de cerchas. Como desventajas se tiene: el alto costo de la placa, alto costo de mano de obra para la instalación de la placa, en la fabricación de la cercha y que la presencia de la placa es poco estética. Por otra parte, se obtiene de las opiniones la recomendación de que, al utilizar la placa, se debe diseñar bajo los datos del fabricante de la misma (cuadro 3). 
Cuadro 2. Distribución de las principales razones para la utilización de cerchas de madera, en Costa Rica, según encuesta de mercado.

Table 2. Distribution of the main reasons for the use of wooden trusses, in Costa Rica, according to market survey.

\begin{tabular}{|cc|}
\hline $\begin{array}{c}\text { Razones para la utilización } \\
\text { de cerchas de madera }\end{array}$ & Porcentaje de respuesta \\
\hline Capacidad soporte del techo & 32,0 \\
\hline Mayor amplitud a la casa & 8,0 \\
\hline Permite mayor ventilación & 4,0 \\
\hline Practica para la construcción & 4,0 \\
\hline Aprovechamiento de & 8,0 \\
\hline caberías & 4,0 \\
\hline Brinda amarre a la estructura & 4,0 \\
\hline Soporte de clavadores & 4,0 \\
\hline Protección & 8,0 \\
\hline Rigidez a la estructura & 8,0 \\
\hline Soporta cargas mayores con & 8,0 \\
\hline menos material & 4,0 \\
\hline Se puede prefabricar & 4,0 \\
\hline Liviana & Barata
\end{tabular}

\section{Percepción de la cercha con placa metálica en el mercado costarricense}

Del total de encuestados un $80 \%$ estaría dispuesto a adquirir la cercha de madera, con uniones de placas metálicas. $Y$ las principales razones del porque la adquirirían y/o utilizarían en sus diseños constructivos serían: por practicidad, innovación, facilidad constructiva y optimización de costos. Además, entre las opiniones aclaran que sí la compraría siempre y cuando lleguen a la construcción para ser instaladas, para ahorrar mano obra. Para el $20 \%$ de los encuestados, que no la adquirirían sería principalmente porque primero deben comparar su precio con otras opciones y por el espacio que quita en el diseño constructivo.

A los encuestados que sí adquirirían la cercha de madera, con unión de conectores de placa metálica dentada, se les consultó por el precio que ellos le darían al producto, esto para tener un sondeo del precio aproximado a solicitar. Para lo cual se les brindaron tres opciones de rangos de precio que van desde los $\$ 35000$ a los $\$ 50$ 000. Donde un $50 \%$ de los encuestados se inclinó por el rango de precio de $\$ 45000$ a $\$ 50000$ (figura 7).
Cuadro 3. Distribución de la opinión de la utilización de conectores de placa metálica dentada en la fabricación de cerchas de madera, en Costa Rica, según encuesta de mercado.

Table 3. Distribution of opinion on use of metal plate connectors in the manufacture of wooden trusses, in Costa Rica, according to market survey.

\begin{tabular}{|cc|}
\hline $\begin{array}{c}\text { Opinión conectores de placa } \\
\text { metálica dentada }\end{array}$ & Porcentaje de respuesta \\
\hline Tecnológica ingenieril & 30,8 \\
\hline Precio alto & 15,4 \\
\hline Mano de obra alta & 15,4 \\
\hline Poco estéticas & 7,7 \\
\hline Diseñar con datos del & fabricante \\
\hline Reduce espacio en \\
fabricación
\end{tabular}

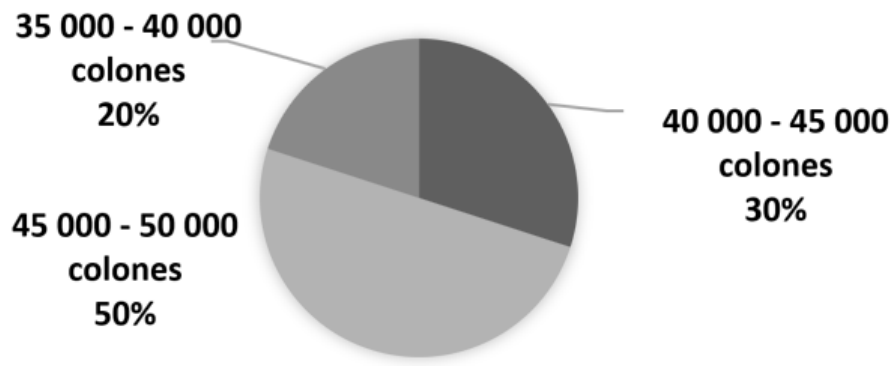

Figura 7. Distribución de rangos de precios para venta de cerchas de madera, con placa metálica dentada, en Costa Rica, según encuesta de mercado.

Figure 7. Distribution of price ranges for sale wooden trusses with metal plates in Costa Rica, according to market survey.

\section{Discusión}

\section{Tipo de profesionales}

Se obtuvo que de los encuestados un $90 \%$, fueron fuentes primarias, es decir, posibles promotores de la utilización de la cercha; y un $10 \%$ de los encuestados corresponde a fuentes secundarias, es decir, sólo un $10 \%$ de los potenciales compradores de la cercha.

\section{Percepción de la madera como material}

La principal razón que demostró la encuesta para construir con madera fue la capacidad de almacenar 
Cuadro 4. Costos por cercha para madera de dos especies: teca y melina.

Table 4. Cost per truss for wood of two species: teak and melina.

\begin{tabular}{|c|c|c|c|c|c|}
\hline Aspecto & Cantidad & Costo unitario colones & Días & Depreciación & Total colones \\
\hline Madera Teca Piezas 5 cmx $10 \mathrm{~cm} \times 250 \mathrm{~cm}$ & 5,5 & 1750 & & & 9625 \\
\hline Madera Teca Piezas $5 \mathrm{~cm} \times 5 \mathrm{~cm} \times 250 \mathrm{~cm}$ & 2,5 & 1750 & & & 4375 \\
\hline Placas & 24 & 300 & & & 7200 \\
\hline Pato Hidráulico * & 1 & 150000 & 1 & 285,1 & 285,1 \\
\hline Herraje * & 1 & 50000 & 1 & 94,7 & 94,7 \\
\hline \multirow[t]{2}{*}{ Mano de Obra } & 2 & 10000 & 1 & & 20000 \\
\hline & & & & Total & 41579,8 \\
\hline Aspecto & Cantidad & Costo unitario colones & Días & Depreciación & Total colones \\
\hline Madera Melina Piezas $5 \mathrm{~cm} \times 10 \mathrm{~cm} \times 250 \mathrm{~cm}$ & 5,5 & 650 & & & 3575 \\
\hline Madera Melina Piezas $5 \mathrm{~cm} \times 5 \mathrm{~cm} \times 250 \mathrm{~cm}$ & 2,5 & 650 & & & 1625 \\
\hline Placas & 24 & 300 & & & 7200 \\
\hline Pato Hidráulico * & 1 & 150000 & 1 & 285,1 & 285,1 \\
\hline Herraje * & 1 & 50000 & 1 & 94,7 & 94,7 \\
\hline \multirow[t]{2}{*}{ Mano de Obra } & 2 & 10000 & 1 & & 20000 \\
\hline & & & & Total & 32779,8 \\
\hline
\end{tabular}

*El pato hidráulico y el herraje, son las herramientas para acoplar las placas metálicas a las piezas de madera. Para estas, se consideró una depreciación de 2 años.

dióxido de carbono $\left(\mathrm{CO}_{2}\right)$. Lo cual también es una de las principales razones para construir con madera en el ámbito mundial. Ramage et al. [24], presentan en estudios recientes del sector de la construcción "que en el Reino Unido con sistemas modulares de marcos de madera pueden ahorrar hasta el $50 \%$ de carbono incorporado y al $35 \%$ de energía incorporada en comparación a los métodos y materiales tradicionales de construcción residencial. Además, la construcción en madera ofrece beneficios que incluyen reducción de desechos, menor costos, y programas de instalación más cortos, lo que significa que estos materiales son cada vez más frecuentes en todo el mercado de la construcción europea".

Todo lo anterior expuesto está relacionado a la segunda razón con mayor cantidad de respuestas: la madera como recurso renovable, donde en las respuestas también involucra los beneficios ambientales, lo cual ha sido de creciente interés y a su vez incentiva la construcción con madera. Así como lo menciona Zalecka [25] las nuevas tendencias de diseño se basan en un desarrollo sostenible de construcción ambiental, que usa edificios naturales donde el material eco-estructural es la madera. Por otra parte, con la misma cantidad de respuestas que por ser un recurso renovable, se encuentra la razón de rápida y fácil construcción. [26] indican que "en una comparación que el tiempo de construcción de una casa de madera es $48 \%$ más corto que para la variante de mampostería. Esto es significativo no solo para el inversor, que puede tener un rendimiento mucho más rápido de sus finanzas, sino también para el medio ambiente. El tiempo más corto de construcción reduce proporcionalmente el impacto medio ambiental con respecto al ruido, polvo y desperdicio".

En tercer puesto por cantidad de respuestas se encuentran: confort, estética, versatilidad de diseños y eficiencia estructural por su alta relación densidad/ resistencia. La madera tiene una baja densidad comparada con materiales como la mampostería y esto resulta en eficiencia para estructuras de largo alcance, en las cuales una parte significativa de la carga que debe transportar la estructura es su propio peso [24].

Por último, otras razones con una sola respuesta cada una son: mejora la temperatura interna en las viviendas, mayor valor agregado (en el caso de consumir madera de plantaciones nacionales) y resistencia a sismos y fuego. Este último respaldado con investigación, donde el Consejo Canadiense de Madera [3], que ha demostrado que edificios con estructura de madera correctamente diseñados logran cumplir con 
los requisitos del código; y por tanto sufren daños limitados por eventos sísmicos y vientos fuertes.

\section{Percepción de la madera de plantaciones forestales por el mercado}

Aunque la cobertura forestal nacional ha aumentado, la demanda de madera producida nacionalmente ha decaído sensiblemente [27]. Lo que se evidencia en lo presentado por la ONF [28], donde menciona "que el $65 \%$ de los productos primarios que se consume en el país, se produce localmente, principalmente madera aserrada. El restante $35 \%$ corresponde a la madera importada, donde el producto principal es la madera aserrada. En el 2011 , el $78,5 \%$ de los productos primarios de madera fueron producidos localmente mientras que en el 2017 fue de $65 \%$, lo que confirma al igual que en años anteriores una contracción en el consumo de los productos primarios de madera producidos en el país". Esto se le atribuye a una serie de dificultades que van desde el aprovechamiento forestal hasta su uso en construcción (Ministerio de Ambiente, Energía y Telecomunicaciones [29]. Otros factores que explican ese decaimiento del uso de la madera, son la falta de especificaciones técnicas como lo tienen otros materiales de la construcción, como el acero o el concreto [16]. Por lo que el Estado enfrenta hoy día, uno de los mayores desafíos ante la oportunidad de aprovechar el potencial de las plantaciones productivas existentes en el país, hacia un producto con un mayor valor agregado [16].

De las opiniones acerca de la madera nacional, la que tuvo mayor cantidad de respuestas fue que la consideran madera de buena calidad para la construcción, lo que coincide con lo mencionado por Paniagua [15] Costa Rica cuenta con madera de calidad constructiva, proveniente de varias especies de plantaciones forestales para abastecer la demanda del mercado. Del volumen total de madera en troza que se procesaron en las industrias de transformación primaria en el 2017, un 21,4 \% se destinó a la industria de la construcción, es decir $209884 \mathrm{~m}^{3}$-r. La mayor parte de la madera se destina a usos de larga duración, mediante los cuales se agrega valor y se mantiene el carbono fijado, generando un beneficio ambiental que otros materiales como el acero, el plástico y el concreto no son capaces de ofrecer [28].

Las especies más conocida según el estudio de mercado fueron las especies de Gmelina arborea (melina) y Tectona grandis (teca). La primera especie probablemente es conocida por su alta tasa de reforestación en Costa Rica [11] y que tiene muchos años de ser usada en los proyectos de reforestación en Costa Rica [30]. En la actualidad se aprovecha casi todo el tronco de esta especie: la parte inferior en la industria del mueble y del contrachapado, la parte intermedia en la construcción, y la parte superior para la fabricación de embalajes [11]. Cabe resaltar que uno de los usos de la G. arborea en la construcción, es para hacer cerchas para casas de habitación [31]. Para el caso de la teca es muy conocida, debido a que es una especie ampliamente comercializada y utilizada por sus características físico-mecánicas, por su buen crecimiento en plantaciones y por la apariencia de su madera [30]. Lo cual la hace una madera versátil para una gran variedad de usos como elementos estructurales [32]. Esta especie, aunque no tiene el conocimiento en el mercado como la madera de teca, nuevamente la cantidad de área plantada y aprovechada hasta el día de hoy hace que esta sea la segunda especie en ser conocida.

Otra de las opiniones, acerca de la madera proveniente de plantaciones forestales, en Costa Rica, que se evidenció a través de la encuesta fue: la poca oferta que presentan los industriales de producir este tipo de madera. Aunque en el pasado la oferta de la madera aserrada para construcción era muy amplio en una variedad de ocho posibles productos. Sin embargo, en la actualidad, y debido a la influencia de la importación de la madera, la oferta de productos ha disminuido notablemente a cinco tipos de productos de madera aserrada de: $2,54 \mathrm{~cm} \times 5,08 \mathrm{~cm}$ (regla 1" $\times 2$ "); $2,54 \mathrm{~cm}$ x 7,62 cm (regla 1 “ x 3"); $5,08 \mathrm{~cm}$ x 10,16 cm (regla 2" x 4"); $5,08 \mathrm{~cm}$ x 15,24 cm (artesón 2" x 6") y 2,54 cm $x 30,48 \mathrm{~cm}$ (formaleta 1" x 12") [12]. Lo cual se refleja en lo presentado por Umaña [10] en una entrevista en proyectos de interés social con madera, donde se explica que el sector constructivo en Costa Rica ha tenido que inclinarse por la madera de exportación, porque no han podido conseguir una oferta que les dé la cantidad de madera necesaria.

De esto nace a su vez la siguiente opinión que es una industria que debe impulsarse, es decir, que la industria en el país es poco tecnificada y/o con poca capacidad de innovación. De acuerdo con Fournier [33], el conocimiento de la madera y el desarrollo de su tecnología en los aspectos de plantación, transformación, tratamientos de secado y preservación, encolados, acabados y otros, podrían garantizar su utilización en la construcción, convirtiendo la madera en el material del futuro. Sin embargo, un problema en nuestro país, es el poco conocimiento que al respecto manejan los ingenieros 
y arquitectos, la escasa información técnica disponible de propiedades y procesamiento de la madera, la poca colaboración entre los actores del sector y la falta de agresividad comercial, son factores que han contribuido al debilitamiento del uso de la madera en la construcción [13], a pesar que en la actualidad se tienen ya disponibles muchas información acerca de las especies de plantación [30].

Y la última opción en cantidad de respuestas es que la madera de plantaciones nacionales no es la idónea para construcción. Esto se debe en gran medida a la falta de información de este material, lo que ha imposibilitado su utilización [16]. Lo que ha generado una falta de bases tecnológicas que repercuten en la falta de reglamentaciones y la no aceptación de este tipo de construcción por no encontrarse en los códigos vigentes [34].

\section{Percepción de la cercha de madera}

Las cerchas de madera son uno de los componentes de las "estructuras livianas" que han sido ampliamente utilizadas a nivel mundial y datan desde el siglo 6to A.C. [35]. Sin embargo, la preferencia del mercado costarricense fue más por vigas y artesones que por la cercha. Según la opinión de un ingeniero civil (uno de los encuestados), la mejor solución para cubiertas siempre dependerá del diseño y de sus condiciones, pero al decir vigas se refiere a piezas sólidas que pueden ser fabricadas de acuerdo a la necesidad tanto en claro como en sección. En tanto, que la preferencia del uso de artesones, se puede deber a la disminución de la oferta de productos de madera aserrada [9]. Por otra parte, de los $209884 \mathrm{~m}^{3}$-r.del volumen total de madera dedicada a la industria de la construcción en Costa Rica, el 14,1 \% se convierte en artesonado, quiere decir madera para uso estructural [28].

Sin embargo, las cerchas tienen la característica de que maximizan la eficiencia estructural, ya que permiten alta rigidez en flexión y alta capacidad de carga, como consecuencia de que la estructura es dividida en un número determinado de piezas, cuyas dimensiones y métodos de unión le conceden niveles de tensión más bajos en comparación con otro tipo de estructuras como las vigas [36].

\section{Conocimiento del mercado acerca de las placas metálicas dentadas}

La unión de las piezas individuales en las cerchas se realiza en puntos concéntricos denominados nudos, en los cuales para su unión comúnmente se ha utilizado clavos y tornillos, en el medio costarricense [14]. Sin embargo, es de conocimiento en países con tradición del uso de la madera como elemento estructural, que con los conectores metálicos tipo placas es posible obtener una mayor resistencia de la madera y por lo tanto de la cercha misma [4].

No obstante, Fueyo et al. [37], mencionan que "desde la invención de la placa metálica dentada, su diseño ha experimentado notables mejoras, en la mayoría de los casos encaminados a ahorrar la mayor cantidad posible de madera. Se estima que los diseños existentes actualmente de placas permiten reducir la cantidad de madera necesaria para construir una estructura entre un 20 y un $30 \%$. Su uso permite la unión de dos o más elementos de la estructura formando un ángulo cualquiera dentro del plano de la misma. El empleo de este tipo de conexiones, presenta ventajas con respecto a otros métodos de unión. Por ejemplo, no condiciona las dimensiones de las piezas a unir. Además, como se emplean en cerchas de pequeñas escuadrías, es posible realizar los ensamblajes en taller, transportando la estructura final entera hasta su posición."

Por otra parte, cabe mencionar que el precio de cada placa ronda entre los 250 a 300 colones (según sus dimensiones) y se utiliza dos placas dispuestas simétricamente a ambos lados del enlace [37], por lo que el precio se puede comparar con la cantidad de clavos o tornillos de las uniones comúnmente utilizadas, además de que estas últimas llevan una pieza de cabería para su unión y con las placas, esta se ahorra.

\section{Percepción de la cercha con placa metálica en el mercado costarricense}

Del $80 \%$ de encuestados que sí adquirirían, se inclinaron por el rango de precio de $\$ 45000$ a $\$ 50$ 000 por cercha. Lo cual, es un precio elevado para el mercado de las cerchas en Costa Rica, ya que se le consultó a los Aserraderos: Maderas S.Q. (Pérez Zeledón, San José, Costa Rica) y Ecocajas (Guápiles, Limón, Costa Rica), ambos dedicados también a la construcción de casas de madera; y el precio unitario por cercha ronda entre 25000 a 40 000 colones, dependiendo de la especie y el tipo de unión (comúnmente clavos o tornillos). Para lo cual se obtuvo un precio por cercha para las dos especies más conocidas, según los encuestados. 


\section{Conclusiones}

El $100 \%$ de los encuestados considera a la madera como un material viable para la construcción. Donde las tres principales razones, para sustentar esta afirmación fueron: la madera almacena dióxido de carbono, por ser un recurso renovable y por ser un material de rápida y fácil construcción. En cuanto, a las respuestas respecto de la madera nacional las opiniones fueron: la madera nacional posee buena calidad, sólo se tiene conocimiento de las especies G. arborea y $T$. grandis; y la falta de capacidad de producción con madera para sufragar al sector constructivo en Costa Rica.

El $28 \%$ de encuestados prefiere la utilización de las cerchas como elemento principal en techumbres. Según la opinión su principal ventaja es: capacidad de soporte del techo. De las personas encuestadas el $90 \%$ conoce del uso de placas metálicas en la construcción de cerchas. De estos el $40 \%$ coincide en que el uso de la placa es una buena opción tecnológica e ingenieril. Finalmente, el $80 \%$ estaría dispuesto a adquirir esta cercha con placa metálica por practicidad, innovación, facilidad constructiva y optimización de costos. Donde el $50 \%$ le daría a esta cercha un rango de precio entre $\$ 45000$ a $\$ 50000$.

\section{Agradecimientos}

Los autores agradecen el apoyo de la Vicerrectoría de Investigación y Extensión del Instituto Tecnológico de Costa Rica.

\section{Referencias}

[1] J. Villar, J. Crespo, J. Lorenzana y M. Guaita, Análisis experimental y por elementos finitos del estado tensional de uniones tradicionales por embarbillado simple y doble en madera estructural. Retos de la Nueva Agricultura Mediterránea, Compostella, 2015.

[2] A. Dykman, «Applying new timber technologies to a variety of multi-unit residential typologies. Studio Pacific Architecture Summer Scholar 2014-2015,» [En línea]. Available: http:// www.studiopacific.co.nz. [Último acceso: 201808 1].

[3] Canadian Wood Council, «Resilient and adaptive design using wood,» 2014. [En línea]. Available: http://cwc.ca/ wp-content/uploads/2017/02/Resilience-Fact-SheetFebruary-8-2017.pdf. [Último acceso: 807 2018].

[4] CORMA (Corporación Chilena de la Madera), Manual: la construcción de viviendas en madera, Santiago Chile. 626 p. : Centro de Transferencia Tecnológica, 2015.

[5] AWC (American Wood Council), Connection solution for wood frame structures, Leesburg. United States of America. 74 p., 2014.

[6] M. Smak y B. Strake, "Development of new types of timber structures based on theoretical analysis and their real behaviour,» Wood Research, vol. 59, n 3, pp. 459-470, 2014.

[7] W. Guo, S. Song, Z. Jiang, G. Wang, Z. Sun, X. Wang, F. Yang, H. Chen, S. Shi y B. Fei, "Effect of metal-plate connector on tension properties of metal-plate connected dahurian larch lumber joints, Journal of Materials Science Research, vol. 3, n 3, pp. 40-47, 2014.

[8] R. Rammer, Wood: Mechanical fasteners. In: saleem hashmi. Reference Module in Materials Science and Materials Engineering, Oxford: 1-10, 2016.

[9] Alpine, «A builders guide to trusses - ebs building supplies. Alpine Systems Corporation,» 2017. [En línea]. Available: http://alpineitw.com/resources/builders-guide-to-trusses.

[10] S. K, Y. Shigemoto, T. Hirai, A. Koizumi y Y. Sasaki, «Shear resistance and failure modes of nailed joints loaded perpendicular to the grain,» Journal Wood Science, vol. 59, p. 255-261, 2013.

[11] R. Moya, "Gmelina arborea in Costa Rica,» Bios et Foréts Des Tropiques, vol. 279, n 1, pp. 47-57, 2004.

[12] C. Tenorio, R. Moya, M. Saenz, A. Navarro, M. Carranza y V. Paniagua, "Diseño, resistencia, tablas de diseño, propuesta de empaque y manuales de uso de cerchas construidas con madera de Gmelina arborea e Hieronyma alchorneoides de plantaciones en Costa Rica,» Revista Forestal Mesoamericana Kurú, vol. 35, n 14, pp. 55-67, 2017.

[13] R. Serrano y R. Moya, «Procesamiento, uso y mercado de la madera en Costa Rica: aspectos históricos y análisis crítico,» Revista Forestal Mesoamericana Kurú, vol. 9, n 21, pp. 1-12, 2011.

[14] R. Moya, «La producción de madera de especies nativas en plantaciones comerciales: una opción real,» Ambientico, vol. 2, n 267, pp. 32-36, 2018.

[15] V. Paniagua, Guía de uso y aplicaciones de la madera en la arquitectura en Costa Rica, Unidad de Investigación de la Escuela de Arquitectura. Universidad de Costa Rica. San José, Costa Rica. 99p, 2010.

[16] A. Coto, Manual de uso de la madera para la construcción. ONF/FONAFIFO, Grupo Nación. San José, Costa Rica. 155p, 2015.

[17] R. Moya, «Industrialización y comercialización de madera proveniente de plantaciones forestales en Costa Rica,» Recursos Naturales y Ambiente, vol. 49, n 50, pp. 154162, 2007.

[18] M. Cruz y R. Puente, «¿Hay verdadera innovación en los lanzamientos de nuevos productos? Factores relevantes de éxito y fracaso en el caso colombiano,» Estudios Gerenciales , vol. 28, pp. 263-280, 201.

[19] W. Pilco y L. Ruiz, Pilco, W. y Ruiz, L. (2015). La investigación de mercados como una disciplina estratégica (Tomo 1), Escuela Superior Politécnica del Chimborazo. Instituto de Investigaciones. Riobamba, Ecuador. 99p. , 2015.

[20] E. Argote, F. Vargas, D. Villada y H. Samuel, «Investigación 
de mercado sobre el grado de aceptación de mermelada de cocona en Sibundoy, Putumayo,» Revista Científica Guillermo de Ockham, vol. 11, n² 2, pp. 197- 206, 2013.

[21] R. Hernández, C. Fernández y P. Baptista, Metodología de la Investigación (Sexta Edición)., Editorial Mac Graw Hill. 634p., 2014.

[22] E. Murgado-Armenteros, M. Gutierrez-Salcedo, F. TorresRuiz y M. Cobo, "Analysing the conceptual evolution of qualitative marketing research through science mapping análisis,» Scientometrics, vol. 102, p. 519-557, 2015.

[23] S. Fleszar, A. Adia, G. Torre, E. Tagai, S. Hall, M. Vira, A. Kutikov, D. Chen, C. Miyamoto, A. Reese, S. Miller, S. Hudson y M. Diefenbach, "Google analytics usage in the study of an online program designed for prostate cancer survivorship," Annals of Behavioral Medicine, vol. 51, n 1 , 2017.

[24] M. Ramage, H. Burridge, M. Busse-Wicher, G. Fereday, T. Reynolds, U. Shah, G. Wu, L. Yu, P. Fleming, D. DensleyTingley, J. Allwood, P. Dupree, P. Linden y O. Scherman, "The wood from the trees: The use of timber in construction," Renewable and Sustainable Energ Reviews, vol. 68, pp. 333-359, 2017.

[25] M. Zalecka, «Unconventional building structures in single family social housing,» Architecture Civil Engineering Environment, vol. 3, pp. 31-36, 2013.

[26] J. Svajlenka, M. Kozlovská y M. Spisáková, «The benefits of modern method of construction based on wood in the context of sustainability,» International Journal of Environmental Science and Technology, vol. 14, p. 15911602, 2017.

[27] ONF (Oficina Nacional Forestal), Informe de usos y aportes 2016, San José. Costa Rica. 40p, 2016.

[28] ONF (Oficina Nacional Forestal), Informe de usos y aportes de la madera en Costa Rica (Estadísticas 2017 y Precios 2018), San José. Costa Rica. 44p, 2018.

[29] E. y. T. MINAE (Ministerio de Ambiente, Estrategia nacional redd+ Costa Rica. Una iniciativa del programa de bosques y desarrollo rural, Borrador para consulta. San José, Costa Rica. 62 p, 2015.

[30] R. Moya y M. Tomazello, «Variation in the wood anatomical structure of Gmelina arborea trees at different ecological conditions in Costa Rica,» Revista Biologia Tropical , vol. 56, n², pp. 689-704, 2008.

[31] J. Vallejos, R. Moya y R. Serrano, «Effects of thinning on diameter, heartwood, density and drying defects of Gmelina arborea,» Maderas.Ciencia y Tecnología, 2015.

[32] R. Keogh, "Las plantaciones de teca en América Latina: mitos y realidades,» Serie Técnica. Boletín Técnico, vol. 397, pp. 8-28, 2013.

[33] R. Fournier, "Construcción sostenible y madera: realidades, mitos y oportunidades, " Tecnología en Marcha, vol. 21, nº 4, pp. 92-101, 2008.

[34] M. Sánchez y C. Mastrandrea, Construcción de viviendas con estructuras de madera plantadas en Costa Rica, Escuela de Ingeniería Forestal. Instituto Tecnológico de Costa Rica. Cartago, Costa Rica. 36p., 2014.

[35] M. Barbari, A. Cavalli, L. Fiorineschi, M. Monti y T. M,
«Innovative connection in wooden trusses,» Construction and Building Materials, vol. 66, pp. 654-663, 2014.

[36] B. Woods, J. Hill y M. Friswell, Ultra-efficient wound composite truss structures, Composites: Part A, 90:111124, 2016.

[37] J. Fueyo, J. Cabezas, M. Dominguez y M. Rubio, «Análisis de tensiones en uniones de tres barras de cerchas ligeras de madera realizadas con placas-clavo,» Información Tecnológica, vol. 206, pp. 45-56, 2009.

\section{Este artículo debe citarse como:}

Rivera-Tenorio, M., Camacho-Cornejo, D., \& Moya, R. (2018). Percepción del mercado costarricense acerca del uso cerchas prefabricadas con madera de plantaciones forestales y unidas con placas metálicas. Revista Forestal Mesoamericana Kurú, 16(38), 35-46. Doi. 10.18845/rfmk. v16i38.3994 